\title{
Mean centred clustering: improving melody classification using time- and frequency-domain supervised clustering
}

\author{
CHANDANPREET KAUR* and RAVI KUMAR \\ Department of Electronics and Computer Engineering, Thapar University, Patiala 147004, India \\ e-mail: lpu_chandan@yahoo.co.in
}

MS received 27 March 2018; revised 13 July 2018; accepted 26 July 2018; published online 1 February 2019

\begin{abstract}
This paper reports a new approach for clustering melodies in audio music collections of both western as well as Indian background and its application to genre classification. A simple yet effective new classification technique called mean centred clustering (MCC) is discussed. The proposed technique maximizes the distance between different clusters and reduces the spread of data in individual clusters. The use of MCC as a preprocessing technique for conventional classifiers like artificial neural network (ANN) and support vector machine (SVM) is also demonstrated. It is observed that the MCC-based classifier outperforms the classifiers based on conventional techniques such as Principal Component Analysis (PCA) and discrete cosine transform (DCT). Extensive simulation results obtained on different data sets of western genre (ISMIR) and classical Indian ragas are used to validate the efficiency of proposed MCC-based clustering algorithm and ANN/SVM classifiers based on MCC. As an additional endeavour, the performance of MCC on preprocessed data from PCA and DCT is studied. Based on simulation results, it is concluded that the application of MCC on DCT coefficients resulted in the highest overall classification success rate over different architectures of the classifiers.
\end{abstract}

Keywords. Artificial neural network; mean centre clustering; musical genre classification; pattern clustering method; support vector machine.

\section{Introduction}

Analysis of melodic structures is a dynamic and rapidly evolving field of research that is enriching the wider signal processing community with exciting applications and challenging problems. This analysis requires retrieval of useful information from a melody, which was traditionally performed by human experts. However, with the advancements in digital signal processing algorithms, machines are used for automatically retrieving the relevant information from music. Based on this extracted information, melodies are clustered into their generic classes. These classes are typically based on the distinct characteristics of a certain group of melodies, which make them particularly suitable for some specific listeners based on their taste of music. Raaga and genre are some examples based on which such clustering can be performed [1].

It is observed from the literature that application of artificial neural network (ANNs) in music analysis in general and melody classification in particular has been rather limited. Nevertheless, ANNs find huge applications in the field of identification, decision making, pattern recognition and clustering. A lot of related work is published in several

*For correspondence studies. A brief survey of these studies is provided in the next section.

\section{Related work}

There have been many works reported in the literature for music classification using different clustering techniques. There are many variants of clustering algorithms, which fall into one of two groups: hierarchical [2] and non-hierarchical [3]. The resulting vectors corresponding to the various files are then classified or clustered using existing classification software, based on various standard statistical pattern recognition classifiers, $k$-prototype algorithm [4], Bayesian classifiers [5], hidden Markov models [6], ensembles of nearest neighbour classifiers or neural networks (NNs). Purohit and Joshi [7] introduced a new efficient approach towards $K$-mean clustering algorithm. They proposed a new method for generating the cluster centre by reducing the mean square error of the final cluster without large increment in the execution time. It reduced the means square error without sacrificing the execution time.

Authors in [8] proposed brain tumour segmentation using $K$-mean clustering and fuzzy $c$-mean algorithm. Yedla et al [9] proposed an enhancing $K$-mean clustering algorithm 
with improved initial centre. A new method for finding the initial centroid is introduced and it provides an effective way of assigning the data points to suitable clusters with reduced time complexity. They proved that their proposed algorithm has more accuracy and needs less computational time compared with the original $K$-mean clustering algorithm. Authors in [10] indicated the ability of clustering to group documents with respect to the topic of relevance; such findings are the basis for the clustering hypothesis. To group a set of documents into clusters of documents relevant to different instances of a topic requires clustering with respect to instance relevance.

Authors in [11] use the Self-Organized Map (SOM) technique and preprocess the data of raw music raga files, from which they utilize the frequency-domain sampling by FFT, which shows highly co-relation with the clusters they are predicted to belong to. In [12], Kirthika and Chattamvelli proposed a general system architecture for clustering and classification purpose of ragas.

In [13], a method of music segmentation is proposed, which is based on hierarchical labelling of spectral features. A method based on strong changes of timbre to indicate possible section boundaries of music was given in [14] and the method proposed in [15] is based on calculating the reoccurrence of sections of a particular type for clustering melodies.

\section{Novel aspects}

Classification of melodic structures poses a plethora of computational challenges due to poor separability of musical data in the pattern space. It is a well known fact that the performance of any classifier depends greatly on the type of data given as input. Therefore, it is quintessential to apply preprocessing to the data before applying classification. This results in linear separability of the data in pattern space. This has served as a motivation for the authors to apply mean centred clustering (MCC) on discrete cosine transform (DCT)-processed data. It is evident from the results that the overall classification percentage improves when MCC is applied on raw data. However, in some cases, the classification percentage is low. To improve this performance, MCC is applied on DCT-processed data. For example, in case of ANN classifier, when the number of hidden layers is increased, the classification percentage improves for the case when MCC is applied on DCT-processed data. Experiments were conducted on other combinations like PCA on MCC but the results were rejected due to poor classification performance of such combinations. Conventional techniques like PCA and DCT lack in creating linear separability, which introduces improper and overlapped decision boundaries.

The novel contribution of this paper is introduction of a clustering technique that centres the training samples of the musical piece about its cluster mean called as MCC. This improves the class separability of the samples in pattern space. This clustering technique has been applied both on the time-domain and frequency-domain samples kept aside for the training phase.

The idea behind the proposed technique is derived from the context of image de-blurring, where it is observed that a clustered pattern space improves the performance of the NN. A clustered pattern space contains different distribution characteristics for different classes of data, which causes the NN to search for a common ground while approximating the underlying function. A network trained this way is less likely to suffer from overfitting and more likely to yield good performance with unlabelled samples [16].

Finally, the use of MCC as a preprocessing technique for conventional classifiers like $\mathrm{ANN}$ and support vector machine (SVM) is also demonstrated. It is observed that the MCC-based classifier outperforms the classifiers based on conventional techniques such as Principal Component Analysis (PCA) and DCT. The efficiency of proposed clustering technique is validated using extensive simulation results.

The remainder of the paper is organized as follows. Section 2 presents the work related to the problem discussed in this article while section 3 presents the novel aspects of the proposed technique. The MCC algorithm is explained in section 4. Genre classification via MCC is presented in section 5. Section 6 gives the details of data acquisition while results and discussion are presented in Section 7. Section 8 concludes the article.

\section{4. $\mathrm{MCC}$}

\subsection{Challenges in melody clustering and classification}

Clustering and classification of melodic structures poses a plethora of computational challenges due to poor separability of musical data in the pattern space. It often becomes necessary to make the data linearly separable in the pattern space before subjecting it to a classifier. Some of the challenges encountered are

- very high dimensionality of the data samples in a single melody file,

- very large size of the databases and

- overlapping boundaries of cluster classes.

It has also been observed that conventional techniques like PCA and DCT lack in creating linear separability, which introduces improper and overlapped decision boundaries. This has served as a motivation for the authors to envisage a technique that could improve the class separability of the data in the pattern space. 


\subsection{Clustering using MCC}

In this subsection, the algorithm of MCC is explained. MCC is a clustering technique based on the first level (mean) and second level (variance) statistics of data. These statistics are very easy to calculate, which makes MCC a highly efficient clustering technique. The proposed technique to centre the cluster on its respective mean is described by a flow chart given in figure 1 .

The melody to be clustered is first represented as a data vector consisting of the samples of melody. Then the mean and variance of this data vector are calculated. Mathematically, for a data vector $d$ containing $n$ samples $d_{1}, d_{2}, \ldots d_{n}$, the mean $S_{d}$ is calculated as

$$
S_{d}=\frac{1}{n} \sum_{i=1}^{n} d_{i}
$$

Now, the variance $V_{d}$ is calculated as

$$
V_{d}=\frac{1}{n} \sum_{i=1}^{n}\left(d_{i}-S_{d}\right)^{2}
$$

Finally the clustering is made based on the value of the variance $V_{d}$ calculated in (2).

In order to cluster the data using MCC, the data vector $d$ needs to be transformed into new data vector $t$. Mathematically, the transformed value is calculated as

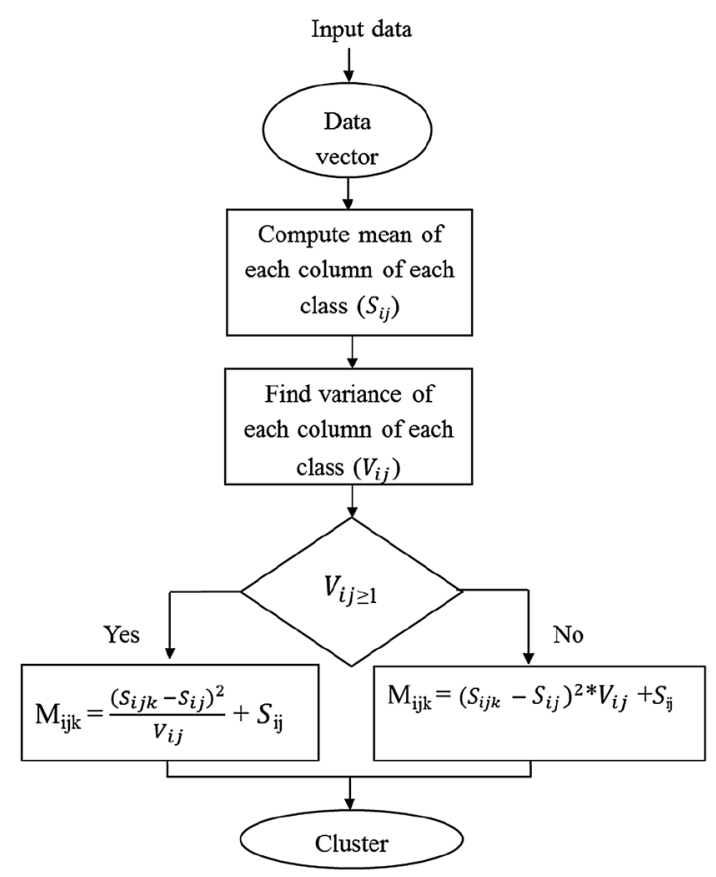

Figure 1. Flow chart to implement the proposed classification system.

$$
\begin{aligned}
t & =\frac{\left(d_{i}-s_{d}\right)^{2}}{V_{d}}+S_{d} \quad \text { for } \quad V_{d} \geq 1 \\
& =\left(d_{i}-s_{d}\right)^{2} V_{d}+S_{d} \quad \text { for } \quad V_{d}<1
\end{aligned}
$$

Since each sample needs to be uniquely centred about the class mean, means of samples from each class are computed for every individual data vector. Similarly, variances are computed as average deviation of each sample of a particular row from its class mean. The transformed values are nothing but variance-normalized deviation of samples from their class mean represented about the mean itself. As a result of this transformation, data are clustered in the pattern space.

\subsection{MCC on DCT}

As the result of any preprocessing technique should be independent of the choice of training and test sets, the authors were motivated to employ DCT prior to the application of MCC. It is well known that cosine transforms map the raw data in time domain to transformed spaces in the frequency domain where the natural clusters in the data become more distinguishable. Therefore, subsequent application of MCC on DCT coefficients is likely to ensure better generalization by final classifiers. DCT coefficients obtained are clustered using MCC.

\section{Genre classification via MCC}

Two popular classifiers: backpropagation-trained ANN and SVM, are employed for the task of classification of melodic structures in this work. It is observed from the literature that application of ANNs in music analysis in general and melody classification in particular has been rather limited. Nevertheless, ANNs find huge applications in the field of identification, decision making, pattern recognition and clustering.

Neural nets are widely used in pattern recognition because of their ability to generalize and to respond to unexpected inputs/patterns. The network can be trained to perceive the criteria used to classify, and it can do so in a generalized manner, allowing successful classification of new inputs that are not used during training [17]. It can work with large numbers of qualitative variables such as behaviours, provided that it can be coded, and it can use non-linear linked variables [18]. On the other hand, SVM is a popular statistically robust learning method based on risk minimization. SVM trains a classifier by finding an optimal separating hyperplane that maximizes the margin between two classes of data in the kernel-induced feature space [19]. From the available literature it is observed that supervised clustering as a pre-processing step results in significant improvement in the training phase performance of ANN/ 
SVM, which may further lead to good generalization in the testing phase [20, 21]. Thus, this method essentially works for new data points with unknown labels after properly training the classifier.

To address the problem of genre classification, we utilize the proposed clustering technique followed by conventional classifiers like SVM and ANN to automatically classify music genre.

\section{Data acquisition and description}

Two different data sets are used in this work to check the validity of proposed technique. A brief description of these two data sets is given below.

\subsection{Data set 1}

The first data set consists of 50 songs of 5 classes from different ragas Asa, Basant, Malhar, Shiri and Gauri, which belong to classical Indian music. Ten songs from each class were considered. We further extracted three clippings from each song. The raga samples have been obtained from www.searchgurbani.com whose excerpts are taken from Guru Granth Sahib: An Advanced Study [22], a book considered to be a scholarly source of authentic information on Indian religious music and poetry.

In the context of Indian classical music, raga is the primary melodic mode. A raga is a tonal framework for composition and characterization. Music historians have found that mediaeval European church modes bear significant similarity to ragas in Indian musical tradition [23]. A raga uses a series of melodic notes upon which a melody is built. Compared with classification of individual melodies, automatic identification of a raga is a bit more challenging task. This is because the way the notes are approached and rendered is more important in defining a raga than the note itself. Furthermore, no two performances of the same raga need be identical even by the same artist. Nevertheless, automatic raga identification can provide a basis for searching of similar songs and generating automated play lists that are suited for a certain aesthetic theme [12, 24].

\subsection{Data set 2}

In order to validate the performance of the proposed technique on musical pieces other than ragas, the same was applied on MIDI ISMIR database Music Audio Benchmark Data Set. Fifty songs of 20-s duration each, three from each genre pop, folk country, electronic and blues have been pooled and named as MIDI Database in this work [25].

\section{Results and discussion}

\subsection{Results from scatter plots}

The raw music files of raga data belonging to Data set 1 are shown as scatter plot in figure 2. Legend shows generic name of the raga classes. For the sake of brevity, scatter plots for only raga data set is shown. However, final classification results will be furnished for both raga and MIDI data sets.

The raw data scatter plot depicts time-domain samples obtained from the imported files. Here, D1, D2 and D3 represent dimensions with the largest, second largest and third largest variance, respectively. Similar scatter plots can be drawn with other combinations of dimensions. However, for the sake of convenience we have chosen to reproduce only figure 2 as a representative plot of raw data in the pattern space. The jumbled nature of raw data is evident from figure 2 and it is necessary to apply an appropriate pre-processing technique before subjecting it to the final classifier.

We compare the proposed clustering algorithm to some other techniques available in literature like PCA and DCT. PCA is a standard statistical technique that can be used to reduce the dimensionality of a data set of different classifiers on different audio feature sets to determine the genre of a given music piece, without much loss of information. PCA is an unsupervised method, which makes no use of information embodied within the class variable. DCT is a useful approach used in signal and image processing. It transforms the signal, from spatial domain into frequency domain. The two-dimensional DCT is calculated by matrix multiplication to reduce the computational complexity by row column decomposition.

The scatter plot against the first three principal component axes obtained from PCA is shown in figure 3. The scatter plot of DCT-processed data is shown in figure 4. DCT and PCA produce overlapped clusters. Hence clustering efficiency is poor. The scatter plot created as a result of MCC on raw data is shown in figure 5. It is evident from figure 5 that the application of MCC has resulted in the improved class separability in a three-dimensional pattern space. To compare the clustering results of MCC with other techniques available in literature like PCA and DCT, we utilize both scatter plots and the Davies-Bouldin (DB) index.

It is not always possible to visually analyse the class separability of the data, especially when it is high dimensional. Therefore, we have employed a cluster validity measure. In this work, the DB index has been employed as the validity measure. This index is a function of the ratio of the sum of within-cluster scatter to between-cluster separation. A lower DB index is indicative of compact and wellseparated clusters and is a measure of effectiveness of underlying clustering technique [26]. 


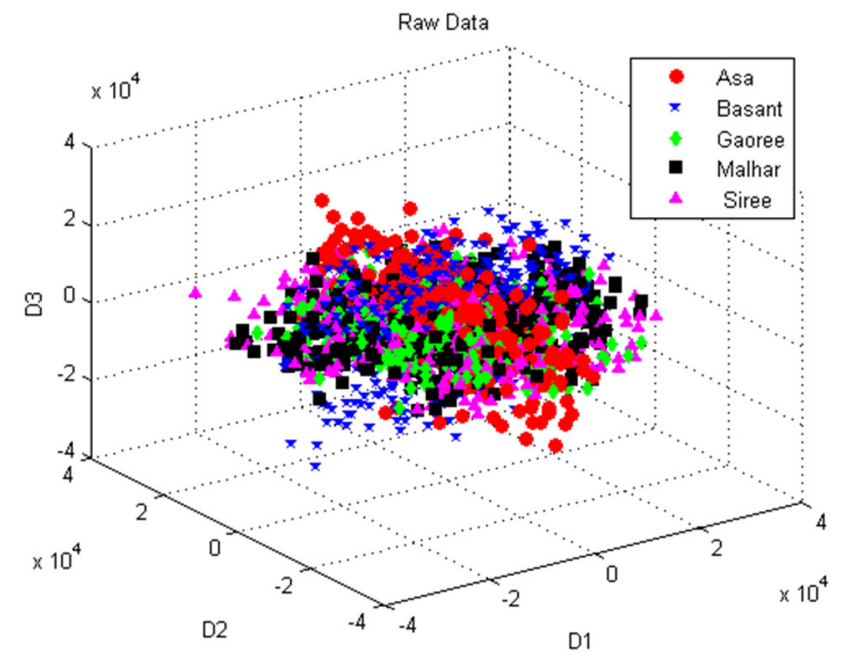

Figure 2. Raw data scatter plot.

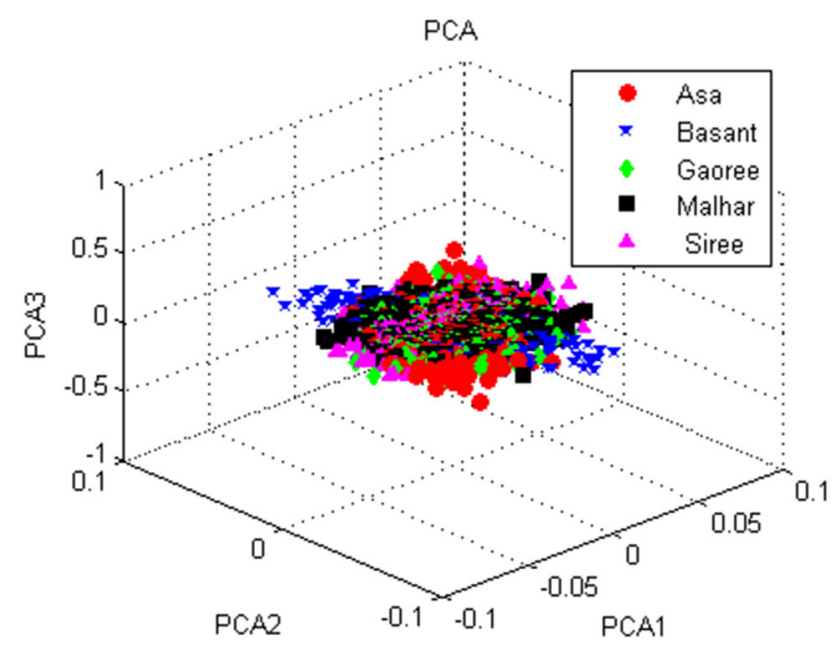

Figure 3. PCA scatter plot.

Let $\sigma_{i}$ be the average distance of each sample in the $i^{\text {th }}$ cluster from its mean. If there are $k$ clusters in the data, the DB index is given by

$$
D B=1 / k \sum_{i=1}^{k} \max \frac{\sigma_{i}+\sigma_{j}}{d\left(C_{i}, C_{j}\right)}, i \neq j
$$

where $d\left(C_{i}, C_{j}\right)$ is the inter-cluster distance between any two cluster centroids $C_{i}$ and $C_{j}$. DB index has been calculated for both the raw and the pre-processed data using Euclidean distance (E.D.) and Mahalanobis distance (M.D.) as metrics.

The index was computed employing both E.D. and M.D. DB indices of clusters formed with data pre-processed using different techniques and different distance metrics have been enlisted in table 1. It can be observed that MCC

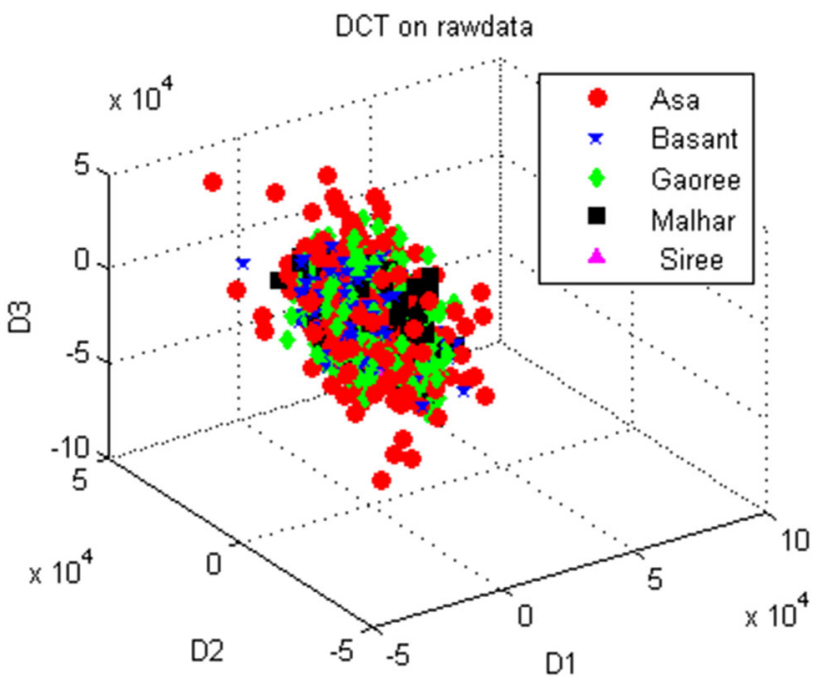

Figure 4. DCT scatter plot.

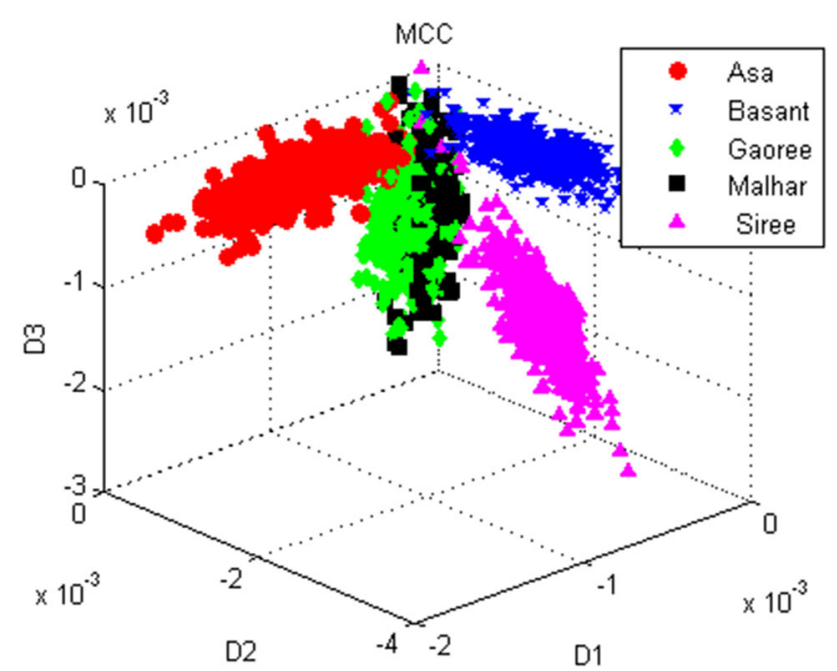

Figure 5. MCC on raw data scatter plot.

applied on DCT coefficients yields the best cluster validity and hence it is expected to be reflected in the final classification success rate. The MCC on DCT scatter plot is shown in figure 6. Furthermore, raga data set has poor cluster validity as compared with MIDI data even after preprocessing. We have compared the results given in [27] in which an Mel-frequency cepstral coefficient (MFCC)-based classifier is used. The work is based on Carnatic Music. We have taken the cases of only the parent ragas for comparison. As given in table 1 of [27], an overall accuracy of about $20-30 \%$ is obtained using the MFCC-based algorithm. However, the use of our proposed MCC-based classifier results in 50\% accuracy. Moreover, an additional $5 \%$, i.e., $55 \%$, accuracy is obtained in classification using DCT-based MCC. 
Table 1. DB indices of clusters formed with training data.

\begin{tabular}{lccccccc}
\hline & & $\begin{array}{c}\text { DB index } \\
\text { (Raga data } \\
\text { set) }\end{array}$ & & \multicolumn{2}{c}{$\begin{array}{c}\text { DB index } \\
\text { (MIDI data } \\
\text { set) }\end{array}$} \\
\cline { 3 - 4 } Sl. no. & Type of data & E.D. & M.D. & & E.D. & M.D. \\
\hline 1 & $\quad$ Raw data & 1.29 & 1.06 & & 0.98 & 0.76 \\
2 & PCA-processed data & 1.22 & 1.03 & & 0.98 & 0.76 \\
3 & DCT coefficients & 1.05 & 1.01 & & 0.87 & 0.66 \\
5 & MCC on raw data & 0.85 & 0.99 & & 0.65 & 0.79 \\
5 & MCC on DCT coefficients & 0.45 & 0.85 & & 0.44 & 0.25 \\
\hline & & & & & &
\end{tabular}

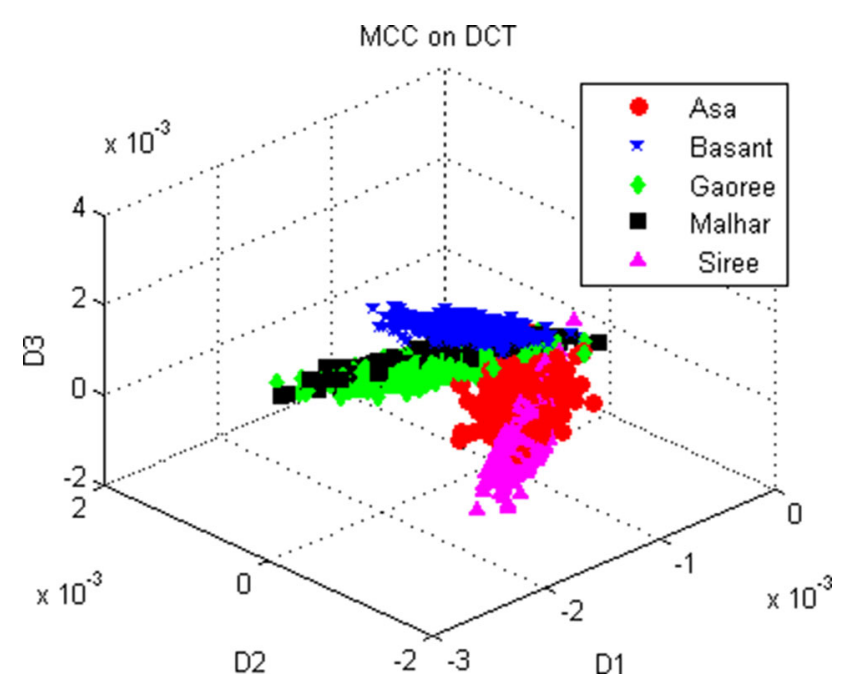

Figure 6. MCC on DCT scatter plot.

It is to be noted that MCC gives unique non-overlapping clusters. DCT on MCC gives even better results. In order to prove this fact, two classifiers have been used and their performance for each case, i.e., with and without MCCprocessed data, is evaluated in the next subsection.

\subsection{ANN and SVM classifiers}

The present work employs backpropagation-algorithmtrained ANN and SVM as final classifiers. Backpropagation algorithm, recognized all over the world as a powerful tool to train multilayer perceptrons, uses gradient descent technique to update the network weights [28]. PCA, DCT and $\mathrm{MCC}$ are used as pre-processing techniques followed by ANN/SVM as a classifier. The block diagram of classification process is shown in figure 7. It may be worth mentioning that the complexity of $\mathrm{NN}$ implementing the global approximation strategy is less as compared with SVM because NN employs very small number of hidden neurons [29, 30]. On the other hand, the SVM is based on

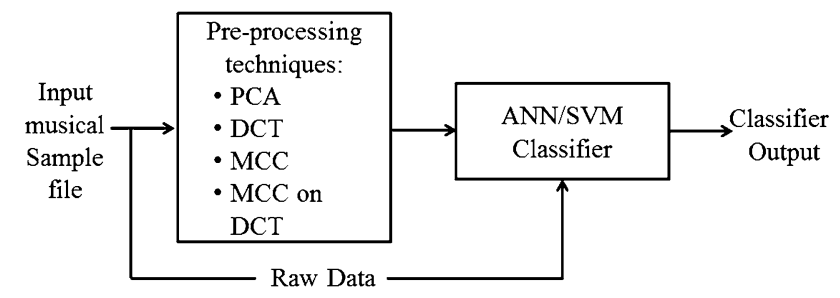

Figure 7. Block diagram of classification system.

Table 2. ANN classification results (raga data).

\begin{tabular}{lccccc}
\hline & \multicolumn{5}{c}{ Percentage classification } \\
\cline { 2 - 6 } & \multicolumn{5}{c}{ Pre-processing (\%) } \\
\cline { 2 - 6 } $\begin{array}{l}\text { No. of neurons in } \\
\text { the hidden layer }\end{array}$ & $\begin{array}{c}\text { Raw } \\
\text { data }\end{array}$ & PCA & DCT & raw data & MCC \\
on DCT \\
\hline 2 & 27.50 & 31.90 & 40 & 53 & 32 \\
3 & 35.80 & 41.60 & 52 & 39.80 & 58.90 \\
4 & 47.80 & 27.50 & 40 & 63.50 & 58.20 \\
5 & 35 & 48 & 47 & 42.70 & 67.40 \\
6 & 42 & 36.60 & 48.60 & 42 & 51.30 \\
7 & 35.50 & 33.90 & 39 & 51 & 57.50 \\
8 & 36.60 & 43 & 46 & 47.80 & 38 \\
\hline
\end{tabular}

Table 3. ANN classification results (MIDI data).

\begin{tabular}{lccccc}
\hline & \multicolumn{5}{c}{ Percentage classification } \\
\cline { 2 - 6 } & \multicolumn{5}{c}{ Pre-processing (\%) } \\
\cline { 2 - 6 } $\begin{array}{l}\text { No. of neurons in } \\
\text { the hidden layer }\end{array}$ & Raw & & & MCC on & MCC \\
data & PCA & DCT & raw data & DCT \\
\hline 2 & 33.50 & 43.50 & 38.20 & 62.00 & 53.90 \\
3 & 40.10 & 40.80 & 40.40 & 73.80 & 56.40 \\
4 & 42.70 & 47.70 & 53.30 & 79.50 & 81.30 \\
5 & 43.60 & 39.90 & 38.30 & 74.90 & 83.10 \\
6 & 49.60 & 44.60 & 37.60 & 82.20 & 78.90 \\
8 & 46.90 & 46.50 & 39.00 & 77.10 & 79.50 \\
\hline
\end{tabular}

the local approximation strategy and uses large number of hidden units. For $N$ number of samples, the computational cost of NN implemented by multi-layer perceptron has been found to be on the order of $N^{2}$ whereas for SVM it approaches $N^{3}$ [31, 32].

For both SVM and ANN, 10-fold cross-validation was used in this work, which divides the data into set of $K$ subsets of the same size. One of the subsets is used as a validation data set in turn to test the model and remaining $K-1$ subsets are combined together for training data set. The cross-validation process is repeated $K$ times [33]. The 
Table 4. SVM binary classification (raga data).

\begin{tabular}{lccccc}
\hline & \multicolumn{5}{c}{ Percentage classification } \\
\cline { 2 - 6 } & \multicolumn{5}{c}{ Pre-processing (\%) } \\
\cline { 2 - 6 } Classes & Raw & MCC on raw & MCC on \\
data & PCA & data & DCT & DCT \\
\hline Asa & 66 & 66 & 99 & 63 & 74 \\
Basant & 81 & 81 & 99 & 60 & 67 \\
Malhar & 54 & 58 & 72 & 52 & 88 \\
Shiri & 59 & 61 & 79 & 74 & 97 \\
Gauri & 62 & 54 & 87 & 63 & 69 \\
\hline
\end{tabular}

Table 5. Confusion matrix of raga data (multi-SVM).

\begin{tabular}{lccccc}
\hline & \multicolumn{5}{c}{ Overall percentage classification: $24.16 \%$ of test } \\
samples \\
\cline { 2 - 6 } Classes & Asa & Basant & Malhar & Shiri & Gauri \\
\hline Asa & 61 & 39 & 32 & 59 & 83 \\
Basant & 49 & 13 & 64 & 75 & 74 \\
Malhar & 56 & 14 & 77 & 54 & 74 \\
Shiri & 58 & 31 & 63 & 57 & 66 \\
Gauri & 76 & 26 & 23 & 26 & 124 \\
\hline
\end{tabular}

classifier was trained to 500 epochs and the training function trainlm was used. The number of neurons in hidden layer was varied experimentally and a particular architecture was trained with input data a number of times. All the architectures were then simulated with the test data and actual classification performance for unlabelled samples was noted.

We have trained the network 20 times each with a particular architecture. The architecture was changed by changing the number of neurons in the hidden layer from two to eight. Since initial weights for each experiment have been saved, testing was performed for each architecture using the weight set that resulted in minimum training error. Tables 2 and 3 report testing phase performance for the ANN classifier. The melodies were taken from raga Asa in the results given in table 2 . In table 3, the MIDI data were taken from the western data set of class POP. It is evident that application of MCC on raw data results in significantly better classification results for almost all the architectures. However, the best classifications were obtained when ANN was trained with MCC-processed DCT coefficients. With five neurons in the hidden layer, $67.4 \%$ success rate has been obtained for the raga data.

Employing MCC on DCT gave better results with MIDI data set, where we obtained $83 \%$ success rate in the testing phase. This may be attributed to simpler structure of features of songs in the MIDI data set. Nevertheless, efficacy
Table 6. Confusion matrix of MCC-processed raga data (multiSVM).

\begin{tabular}{|c|c|c|c|c|c|}
\hline \multirow[b]{2}{*}{ Classes } & \multicolumn{5}{|c|}{$\begin{array}{l}\text { Overall percentage classification: } 92.43 \% \text { of test } \\
\text { samples }\end{array}$} \\
\hline & Asa & Basant & Malhar & Shiri & Gauri \\
\hline Asa & 270 & 1 & 2 & 0 & 1 \\
\hline Basant & 0 & 239 & 36 & 0 & 0 \\
\hline Malhar & 4 & 48 & 223 & 0 & 0 \\
\hline Shiri & 6 & 2 & 3 & 264 & 0 \\
\hline Gauri & 0 & 1 & 0 & 0 & 274 \\
\hline
\end{tabular}

Table 7. SVM binary classification (MIDI data).

\begin{tabular}{|c|c|c|c|c|c|}
\hline \multirow[b]{3}{*}{ Classes } & \multicolumn{5}{|c|}{ Percentage classification } \\
\hline & \multicolumn{5}{|c|}{ Pre-processing (\%) } \\
\hline & $\begin{array}{r}\text { Raw } \\
\text { data }\end{array}$ & PCA & DCT & $\begin{array}{c}\text { MCC on raw } \\
\text { data }\end{array}$ & $\begin{array}{c}\text { MCC on } \\
\text { DCT }\end{array}$ \\
\hline Pop & 69 & 69 & 38 & 97 & 76 \\
\hline $\begin{array}{l}\text { Folk } \\
\text { Country }\end{array}$ & 47 & 50 & 37 & 90 & 92 \\
\hline Electrics & 51 & 53 & 51 & 89 & 50 \\
\hline Blues & 55 & 64 & 59 & 79 & 76 \\
\hline Alternative & 41 & 48 & 53 & 99 & 94 \\
\hline
\end{tabular}

Table 8. Confusion matrix of multi-SVM on MIDI raw data.

\begin{tabular}{lccccc}
\hline & \multicolumn{5}{c}{$\begin{array}{c}\text { Overall percentage classification: } 29.39 \% \text { of test } \\
\text { samples }\end{array}$} \\
\cline { 2 - 6 } Classes & Pop & $\begin{array}{c}\text { Folk } \\
\text { Country }\end{array}$ & Electrics & Blues & Alternative \\
\hline Pop & 45 & 18 & 25 & 22 & 109 \\
Folk & 7 & 40 & 57 & 8 & 108 \\
$\quad$ Country & & & & & \\
Electrics & 6 & 56 & 49 & 19 & 90 \\
Blues & 13 & 44 & 42 & 25 & 96 \\
Alternative & 27 & 14 & 8 & 7 & 164 \\
\hline
\end{tabular}

of MCC as a pre-processing stage may be deemed established. Tables 4, 5, 6, 7 and 8 present results of SVM classification both in binary and multiclass modes. Tables 4 and 6 show results in terms of percentage classification of test samples when the training samples were processed using different techniques, while tables 5 and 7 are confusion matrices showing number of test samples correctly assigned to their respective classes. A close inspection of tables 5 and 7 reveals that MCC-processed data dramatically improve the multi-class generalization performance of the SVM classifier. Comparison of traditional techniques, 
Table 9. Confusion matrix of multi-SVM on MIDI MCC-processed data.

\begin{tabular}{lccccc}
\hline & \multicolumn{5}{c}{ Overall percentage classification: $93.27 \%$ of test } \\
samples \\
\cline { 2 - 6 } Classes & Pop & Country & Electrics & Blues & Alternative \\
\hline Pop & 218 & 0 & 0 & 1 & 0 \\
Folk & 0 & 194 & 26 & 0 & 1 \\
$\quad$ Country & & & & & \\
Electrics & 2 & 30 & 188 & 0 & 0 \\
Blues & 7 & 0 & 8 & 205 & 0 \\
Alternative & 0 & 0 & 0 & 0 & 220 \\
\hline
\end{tabular}

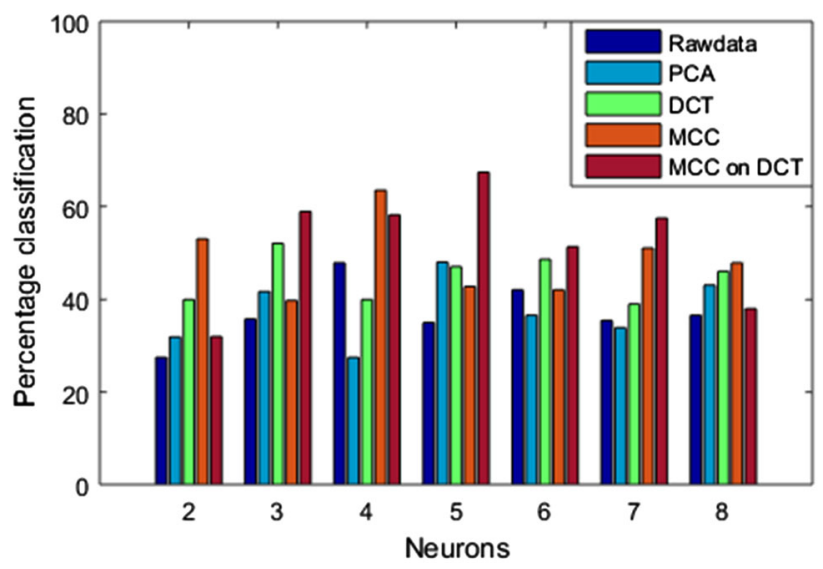

Figure 8. Performance comparison of preprocessing techniques on raga data (ANN classifier).

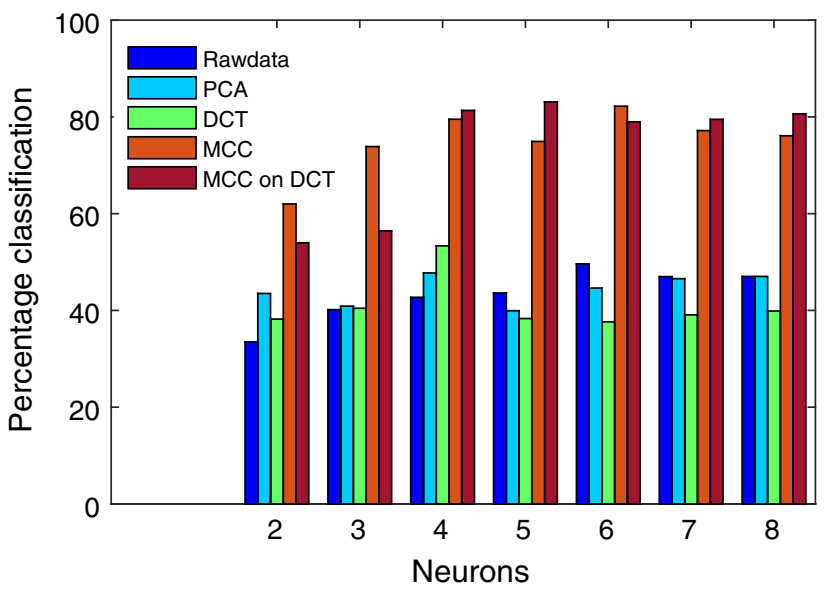

Figure 9. Performance comparison of preprocessing techniques on MIDI data (ANN classifier).

viz., PCA and DCT, with the proposed MCC is provided in figures 8 and 9 for different ANN architectures. Tables 8 and 9 show confusion matrix of multi SVM on MIDI raw data and MCC process data respectively. It is evident that MCC on DCT on raga data yields the best classification result of $67.4 \%$ and on MIDI data, $83.1 \%$ unlabelled samples were correctly assigned to their parent class. It is observed that in case of SVM classifier, applying DCT before MCC does not improve the performance. The reason for such results may be that DCT in this case is not able to provide linear separability of the data in pattern space. Instead, it makes the process more complex, which results in deterioration of the results.

\section{Conclusion}

This work demonstrates the efficacy of supervised clustering on the final classification of musical signals. The application of MCC on both raw data and DCT coefficients has served to improve the classification success rate substantially. Furthermore, performance of the ANN classifier has been more consistent with the MCC-processed data than with raw data, PCA-processed data or with DCT coefficients. The proposed technique has given even better results with the SVM classifier. The correlation between a lower DB index of the preprocessed clusters and the percentage classification obtained using ANN/SVM classifiers underscores the necessity of cluster validity measures in the selection of an appropriate pre-processing technique. Future work may be directed towards making the pre-processing stage unsupervised so that we do not have to rely on a large pool of data for training. It is also hoped that identification/ extraction of other appropriate features from musical samples may further enhance the success rate of the final classifier.

\section{References}

[1] Muller M, Ellis D P, Klapuri A and Richard G 2011 Signal processing for music analysis. IEEE Journal of Selected Topics in Signal Processing 5(6): 1088-1110

[2] Htike K K 2018 Forests of unstable hierarchical clusters for pattern classification. Soft Computing 22(5): 1711-1718

[3] Frakes W B and Baeza Yates R (Eds) 1992 Information retrieval: data structures \& algorithms. Englewood Cliffs, New Jersey: Prentice-Hall

[4] Sangam R S and Om H 2018 An equi-biased k-prototypes algorithm for clustering mixed-type data. Sadhana 43(3): 37

[5] Seltzer M L, Raj B and Stern R M 2004 A Bayesian classifier for spectrographic mask estimation for missing feature speech recognition. Speech Communication 43(4): 379-393

[6] Rabiner L R 1989 A tutorial on hidden Markov models and selected applications in speech recognition. Proceedings of the IEEE 77(2): 257-286

[7] Purohit P and Joshi R 2013 A new efficient approach towards $\mathrm{K}$-means clustering algorithm. International Journal of Computer Applications 65(11): 7-10

[8] Jose A, Ravi S and Sambath M 2014 Brain tumor segmentation using k-means clustering and fuzzy c-means algorithms and its area calculation. International Journal of 
Innovative Research in Computer and Communication Engineering 2(3): 3496-3501

[9] Yedla M, Pathakota S R and Srinivasa T M 2010 Enhancing $\mathrm{K}$-means clustering algorithm with improved initial center. International Journal of Computer Science and Information Technologies 1(2): 121-125

[10] Daisy V R and Nirmala S 2018 Stability-integrated fuzzy C means segmentation for spatial incorporated automation of number of clusters. Sadhana 43(3): 40

[11] Sharma A K, Lakhtaria K I, Panwar A and Vishwakarma S 2014 An analytical approach based on self organized maps (SOM) in Indian classical music raga clustering. In: Proceedings of the Seventh International Conference on Contemporary Computing (IC3), IEEE, pp. 449-453

[12] Kirthika P and Chattamvelli R 2012 A review of raga based music classification and music information retrieval (MIR). In: Proceedings of the IEEE International Conference on Engineering Education: Innovative Practices and Future Trends (AICERA), pp. 1-5

[13] Levy M and Sandler M 2008 Structural segmentation of musical audio by constrained clustering. IEEE Transactions on Audio, Speech, and Language Processing 16(2): 318-326

[14] Foote J 2000 Automatic audio segmentation using a measure of audio novelty. In: Proceedings of the IEEE International Conference on Multimedia and Expo, vol. 1, pp. 452-455

[15] Maddage N C, Xu C, Kankanhalli M S and Shao X 2004 Content-based music structure analysis with applications to music semantics understanding. In: Proceedings of the 12th Annual ACM International Conference on Multimedia, pp. 112-119

[16] Kunz D 2008 An orientation-selective orthogonal lapped transform. IEEE Transactions on Image Processing 17(8): 1313-1322

[17] Schubert E, Canazza S, De Poli G and Roda A 2017 Algorithms can mimic human piano performance: the deep blues of music. Journal of New Music Research 46(2): 175-186

[18] Lek S, Delacoste M, Baran P, Dimopoulos I, Lauga J and Aulagnier S 1996 Application of neural networks to modelling nonlinear relationships in ecology. Ecological Modelling 90(1): 39-52

[19] Vapnik V 1998 Statistical learning theory. New York: Wiley
[20] Harris T 2015 Credit scoring using the clustered support vector machine. Expert Systems with Applications 42(2): $741-750$

[21] Nawi N M, Atomi W H and Rehman M Z 2013 The effect of data pre-processing on optimized training of artificial neural networks. Procedia Technology 11: 32-39

[22] Kapoor S S 2005 Guru Granth Sahib—an advanced study, vol. I. New Delhi: Hemkunt Press

[23] Benward B 2014 Music in theory and practice, vol. 1. New York: McGraw-Hill Higher Education

[24] Sudha R, Kathirvel A and Sundaram R D 2009 A system of tool for identifying ragas using MIDI. In: Proceedings of the Second International Conference on Computer and Electrical Engineering, ICCEE'09, vol. 2, pp. 644-647

[25] Homburg H, Mierswa I, Moller B, Morik K and Wurst M 2005 A benchmark dataset for audio classification and clustering. In: Proceedings of ISMIR 2005, pp. 528-531

[26] Davies D L and Bouldin D W 1979 A cluster separation measure. IEEE Transactions on Pattern Analysis and Machine Intelligence 1(2): 224-227

[27] Daniel H and Revathi A 2015 Raga identification of carnatic music using iterative clustering approach. In: Proceedings of the International Conference on Computing and Communications Technologies (ICCCT), IEEE, pp. 19-24

[28] Haykin S S 2009 Neural networks and learning machines, 3rd ed. Upper Saddle River, NJ, USA: Pearson

[29] Suthaharan S 2016 Machine learning models and algorithms for big data classification. Boston: Springer

[30] Iosifidis A and Gabbouj M 2016 Multi-class support vector machine classifiers using intrinsic and penalty graphs. Pattern Recognition 55: 231-246

[31] Bordes A, Ertekin S, Weston J and Bottou L 2005 Fast kernel classifiers with online and active learning. Journal of Machine Learning Research 6: 1579-1619

[32] Zanaty E A 2012 Support vector machines (SVMs) versus multilayer perception (MLP) in data classification. Egyptian Informatics Journal 13(3): 177-183

[33] Bergmeir C and Benitez J M 2012 On the use of crossvalidation for time series predictor evaluation. Information Sciences 191: 192-213 